\title{
Current Status of Adverse Events Related with Opioid Analgesics in Japan: Assessment Based on Japanese Adverse Drug Event Report Database
}

\author{
Yukio Suga, ${ }^{* a, b}$ Mayako Uchida, ${ }^{b, c}$ Shinya Suzuki, ${ }^{b, d}$ Hideki Sugawara, ${ }^{b, e}$ Kazuhiro Torigoe, ${ }^{b, f}$ \\ Akihiko Futamura, ${ }^{b, g}$ Yoshihiro Uesawa, ${ }^{b, h}$ Takayuki Nakagawa, ${ }^{b, i}$ and Hisamitsu Takase ${ }^{b, j}$ \\ ${ }^{a}$ Department of Clinical Drug Informatics, Faculty of Pharmacy, Institute of Medical, Pharmaceutical \& Health \\ Science, Kanazawa University; 13-1 Takaramachi, Kanazawa, Ishikawa 920-8641, Japan: ${ }^{b}$ Research Promotion \\ Committee, Japanese Society for Pharmaceutical Palliative Care and Sciences; Osaka 550-0001, Japan: \\ ${ }^{c}$ Education and Research Center for Clinical Pharmacy, Osaka University of Pharmaceutical Sciences; 4-20-1 \\ Nasahara, Takatsuki, Osaka 569-1094, Japan: ${ }^{d}$ Department of Pharmacy, National Cancer Center Hospital East; \\ 6-5-1 Kashiwanoha, Kashiwa, Chiba 277-8577, Japan: ${ }^{e}$ Department of Clinical Pharmacy and Pharmacology, \\ Kagoshima University Hospital; 8-35-1 Sakuragaoka, Kagoshima 890-8520, Japan: ${ }^{f}$ Division of Applied \\ Pharmaceutical Education and Research, Hoshi University; 2-4-41 Ebara, Shinagawa-ku, Tokyo 142-8501, Japan: \\ ${ }^{g}$ Department of Pharmacy, Fujita Health University Nanakuri Memorial Hospital; 424-1 Tsu, Mie 514-1295, Japan: \\ ${ }^{h}$ Department of Medical Molecular Informatics, Meiji Pharmaceutical University; 2-522-1 Noshio, Kiyose, Tokyo \\ 204-8588, Japan: ${ }^{i}$ Department of Clinical Pharmacology and Therapeutics, Kyoto University Hospital; 54 Shogoin- \\ Kawahara-cho, Sakyo-ku, Kyoto 606-8507, Japan: and ${ }^{j}$ Nippon Medical School Tama-Nagayama Hospital; 1-7-1 \\ Nagayama, Tama, Tokyo 206-8512, Japan.
}

Received December 19, 2018; accepted February 18, 2019

Opioid analgesics have greatly contributed to the advancement of pain management. However, although opioids have been appropriately used in Japan, they rarely induce serious adverse events, such as respiratory depression. The present study aimed to investigate the temporal changes in the occurrence of opioid-related adverse events and deaths between 2004 and 2017 in Japan using the Japanese Adverse Drug Event Report (JADER) database. We analyzed the following points using data extracted from JADER website: 1) temporal changes in the number and proportion of opioid-related adverse event reports; 2) temporal changes in the number of morphine-, oxycodone-, and fentanyl-related adverse event reports per annual consumption; and 3) cases in which the reported outcome following opioid-related adverse events was death. Our results showed no dramatic changes in the overall incidence of opioid-related adverse events, despite the temporal changes in the annual consumption and shared component of each opioid during the survey period. However, the number and rate of fentanyl-related adverse events and their outcome "death" increased since 2010, being the highest among all adverse event including those related to morphine and oxycodone. Outcome "death" by fentanyl-related adverse events was caused mainly due to respiratory depression. These findings suggest that, although opioid-related adverse events can be controlled through proper monitoring and management by medical personnel in Japan, extra caution should be continuously paid for the rare but serious fentanylinduced adverse events. death

Key words opioid analgesic; Japanese Adverse Drug Event Report; adverse event; proper use; opioid-related

\section{INTRODUCTION}

Since WHO published the first edition of "Cancer Pain Relief" in 1986, the "WHO method of cancer pain relief" has become widespread worldwide. In Japan, extended-release morphine preparations began to be marketed in 1989, while transdermal fentanyl patches and oxycodone extended-release tablets were marketed in 2002 and 2003, respectively. Moreover, methadone tablet and tapentadol extended-release tablets were marketed in 2013 and 2014, respectively. These opioid analgesics have greatly contributed to the advancement of pain management. However, in addition to their general adverse event (AE)s, including constipation, nausea, drowsiness, and delirium, opioids rarely induce serious outcomes, such as respiratory depression and death. ${ }^{1)}$ In the U.S., opioid-related abuse and deaths strikingly increased in the first decade of the 2000 s. $^{2-5)}$ It is reported that opioid-related deaths have risen to
$58 \%$ of all medication-related deaths. $\left.{ }^{6}\right)$ This is because opioids prescribed for family or friends are often used by oneself or because individuals decide to alter the route of administration from oral to self-injection or inhalation by their own judgement.

According to a report comparing the actual consumption of opioid analgesics with the calculated requirement for each country, the adequate rate of opioid use in Japan is $15.5 \%$, which is remarkably low compared to $229.6 \%$ in the U.S., $73.5 \%$ in France, and $66.3 \%$ in the U.K. ${ }^{7)}$ However, with the recent decision to expand opioid indications to include noncancer chronic pain, the yearly consumption is predicted to increase. In Japan, non-cancer chronic pain treatment was added as an indication of the transdermal fentanyl patch in 2010. In the U.S., which preceded Japan in the use of opioids for the treatment of non-cancer chronic pain, opioid-related deaths have become a large societal problem. ${ }^{8)}$ Even in Japan, there 
is concern that the number of AE-related deaths may increase due to the inappropriate use of opioids. For this reason, various types of training for physicians, nurses, and pharmacists are being implemented, in order to promote appropriate opioid use. In 2009, the Japanese Society for Pharmaceutical Palliative Care and Sciences began a system for certifying pharmacists in palliative pharmacotherapy and has been working on educating pharmacists. It is hoped that the training courses offered by relevant academic societies and the activities of palliative field-certified pharmacists will promote appropriate opioid use in Japan. However, since it is difficult to evaluate the outcome of promoting appropriate opioid use, the current status of appropriate use in Japan has not been monitored.

Information for all cases of medical product-induced AEs reported in Japan is publicly available in the "Japanese Adverse Drug Event Report (JADER)" database on the website of Pharmaceuticals and Medical Devices Agency (PMDA) since April 2004. Many cases of opioid-related AEs and their outcomes have been accumulated in this database. Therefore, to assess the current status of appropriate use of opioids and elucidate any problems in promoting appropriate use in Japan, we examined the temporal changes in opioid-related AEs and outcome of "deaths" based on the case reports collected in JADER.

\section{MATERIALS AND METHODS}

Data Selection of Suspected Opioid-Related AEs from the JADER Database JADER AE reports were obtained from the PMDA website (http://www.pmda.go.jp/safety/infoservices/drugs/adr-info/suspected-adr/0003.html). JADER data includes 464259 AE cases recorded between April 2004 and March 2017. In this study, we used data recorded during this period, and analyzed AE reports related with five strong opioids approved in Japan: morphine, oxycodone, fentanyl, methadone, and tapentadol.

In JADER, data for each AE case are divided according to their type and are organized into four tables: a "DEMO table," "drug information table," "REAC table," and a "medical history table." We first removed duplicate data from the "drug information" and "REAC" tables using Hirooka's method.9) Then, we used the identification number of each AE case to merge corresponding case data from the "drug information," "REAC" and "medical history" tables. In this study, we retrieved data for cases with a "suspected medicine." Then, to retrieve cases in which the purpose of administration of opioids was pain management, we selected those that included the word "pain" in the purpose for medication use. ${ }^{10)} \mathrm{We}$ also retrieved cases in which the outcome in opioid-related AE reports was "death." Finally, we transformed the generated data into a data table for use in our analysis.

Data and Statistical Analysis To compare the number of AE reports regarding morphine, oxycodone, and fentanyl according to their consumption, we converted consumption of oxycodone or fentanyl to their morphine equivalent doses. We defined the analgesic activity of oxycodone and fentanyl as 1.5 and 100 times that of morphine, respectively. ${ }^{11)}$ We obtained the data for the annual consumption of the three opioids from CANCER STATISTICS IN JAPAN 2017 ${ }^{11)}$ and normalized the number of AE reports for each drug per year for the period between January 2005 and December 2016 to its morphine
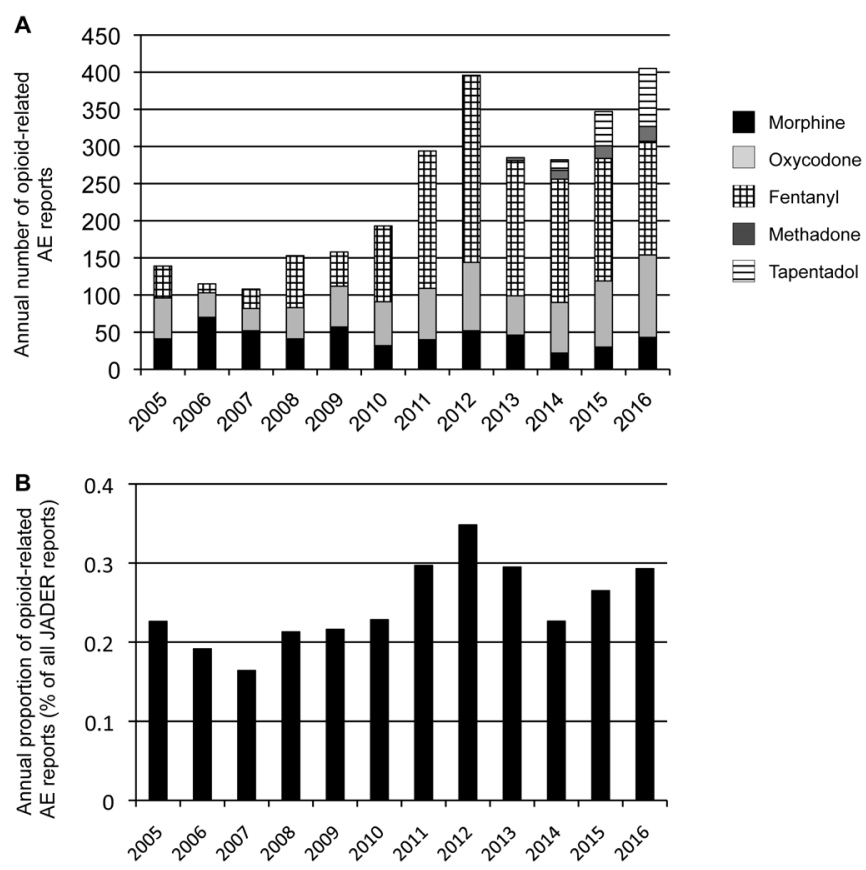

Fig. 1. Temporal Changes in Opioid-Related Adverse Event (AE) Reports in JADER between 2005 and 2016

(A) Annual number of AE reports related with morphine, oxycodone, fentanyl, methadone, and tapentadol. (B) Annual proportion (\%) of opioid-related AE reports among AE reports for all medications included in JADER.

equivalent annual consumption.

For analysis of opioid-related deaths, we used the analysis data table and constructed $2 \times 2$ tables based on two classifications: the presence or absence of the outcome "death" and the presence or absence of the suspected medicine. Then, we calculated the $p$ value by Fisher's exact test, as well as the reporting odds ratio (ROR) and 95\% confidence interval (CI) for opioid analgesics. A $p$ value of $<0.05$ was considered significant. All analyses were performed with $\mathrm{JMP}^{\circledR}$ pro13 (SAS Institute Inc. NC, U.S.A).

\section{RESULTS}

Changes in the Annual Number of Opioid-Related AE Reports The total number of opioid-related AE reports between April 2004 and March 2017 was 3042. We retrieved AE reports for morphine, oxycodone, fentanyl, methadone, and tapentadol from the analysis data table and calculated the number of reports per year for each drug between 2005 and 2016 (Fig. 1A). Although the overall number per year was constant until 2009, it started increasing from 2010 onwards. Regarding the trend of each opioid, the number of morphine-related AE reports gradually decreased, that of oxycodone-related AE reports slightly increased, while that of fentanyl markedly increased since 2010. Methadone AE reports appeared in 2013, but the number was low, while tapentadol reports started increasing after the drug became available in the clinical setting in 2014.

It is noted that the total annual number of AE reports for all medications in JADER was also increased during this period. Therefore, we normalized the annual number of opioid-related AE reports to that of JADER reports for each year (Fig. 1B). The maximum percentage of opioid-related AE reports among all JADER reports was $0.34 \%$ in 2012 and hovered around 
Table 1. Ranking of Reported Opioid-Related Adverse Events (AEs)

\begin{tabular}{|c|c|c|c|c|c|c|c|c|c|c|c|c|}
\hline \multirow[t]{2}{*}{ AEs } & \multicolumn{2}{|c|}{ All opioids } & \multicolumn{2}{|c|}{ Morphine } & \multicolumn{2}{|c|}{ Oxycodone } & \multicolumn{2}{|c|}{ Fentanyl } & \multicolumn{2}{|c|}{ Methadone } & \multicolumn{2}{|c|}{ Tapentadol } \\
\hline & Number & $\% *$ & Number & $\% *$ & Number & $\% *$ & Number & $\% *$ & Number & $\% *$ & Number & $\% *$ \\
\hline Delirium & 236 & 7.8 & 44 & 7.6 & 74 & 9.4 & 85 & 5.7 & 7 & 13.0 & 26 & 18.6 \\
\hline Respiratory depression & 149 & 4.9 & 34 & 5.9 & 29 & 3.7 & 82 & 5.5 & 3 & 5.7 & 1 & 0.7 \\
\hline Nausea & 114 & 3.7 & 27 & 4.7 & 38 & 4.8 & 44 & 3.0 & 0 & 0 & 5 & 3.6 \\
\hline Vomiting & 102 & 3.4 & 22 & 3.8 & 37 & 4.7 & 39 & 2.6 & 0 & 0 & 4 & 2.9 \\
\hline $\begin{array}{l}\text { Altered state of con- } \\
\text { sciousness }\end{array}$ & 87 & 2.9 & 20 & 3.5 & 16 & 2.0 & 46 & 3.1 & 3 & 5.7 & 2 & 1.4 \\
\hline Somnolence & 79 & 2.6 & 19 & 3.3 & 21 & 2.7 & 33 & 2.2 & 5 & 9.4 & 1 & 0.7 \\
\hline $\begin{array}{l}\text { Decreased conscious- } \\
\text { ness level }\end{array}$ & 67 & 2.2 & 15 & 2.6 & 5 & 0.6 & 45 & 3.0 & 0 & 0 & 2 & 1.4 \\
\hline Appetite loss & 55 & 1.8 & 13 & 2.2 & 29 & 3.7 & 13 & 0.9 & 0 & 0 & 0 & 0 \\
\hline Constipation & 49 & 1.6 & 13 & 2.2 & 20 & 2.5 & 10 & 0.7 & 1 & 1.9 & 5 & 3.6 \\
\hline Ileus & 39 & 1.3 & 4 & 0.7 & 21 & 2.7 & 5 & 0.3 & 0 & 0 & 9 & 6.4 \\
\hline All AEs & 3042 & 100 & 579 & 100 & 789 & 100 & 1481 & 100 & 53 & 100 & 140 & 100 \\
\hline
\end{tabular}

* Percentages indicate the number of each AE to the number of all AEs related with the specific opioid.

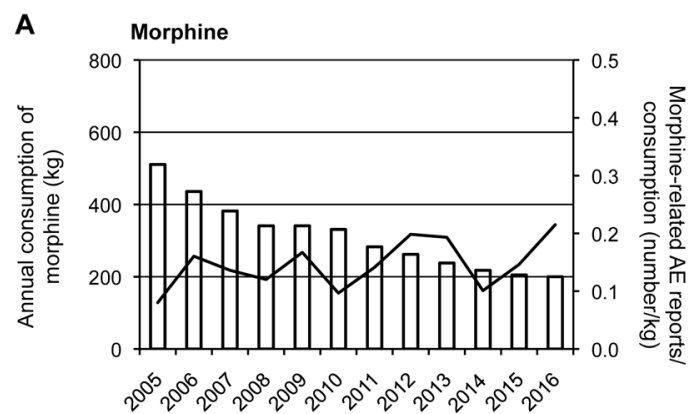

C Fentanyl

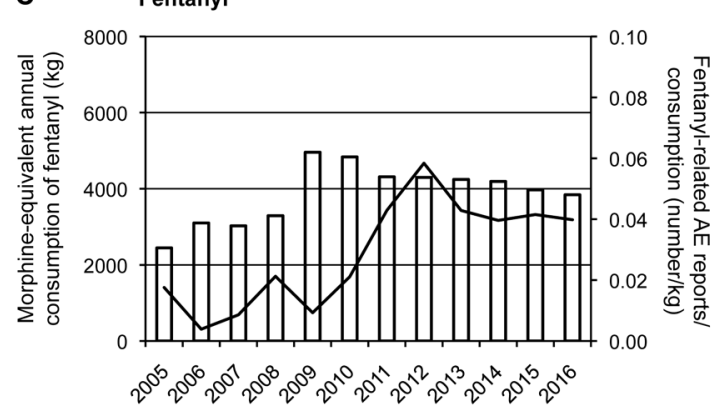

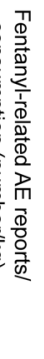

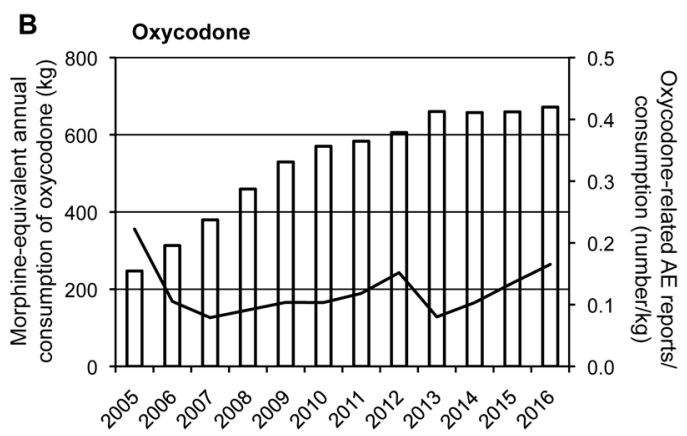

Fig. 2. Changes in the Annual Number of Opioid-Related Adverse Event (AE) Reports According to Consumption between 2005 and 2016

(A-C) Graphs showing the annual consumption (in morphine-equivalent doses, kg; left $y$-axis, bar graph) of morphine (A), oxycodone (B), and fentanyl (C), and the corresponding number of AE reports according to the annual consumption (right $y$-axis, line graph) for each year. Data for the annual consumption of morphine, oxycodone, and fentanyl were obtained from CANCER STATISTICS IN JAPAN 2017.11

\section{$0.2-0.3 \%$ during the survey period.}

We show the top ten AEs with the highest number of reports in Table 1. The most frequent AE was "delirium" in $7.8 \%(236 / 3042)$ of the reports. The second most frequent AE was "respiratory depression" in $4.9 \%$ (149/3042) of the reports, which also ranked within the top three AEs in morphine-, fentanyl-, and methadone-related reports. Other AEs in more than $3 \%$ of the reports were "nausea" $(3.7 \%$; 114/3042) and "vomiting" (3.4\%; 102/3042).

Changes in the Annual Number of Opioid-Related AE Reports According to Consumption We compared the changes in the annual number of morphine-, oxycodone-, and fentanyl-related AE reports according to their annual consumption (in $\mathrm{kg}$ ), after converting consumption of each drug to morphine equivalent doses (Fig. 2). The annual consump- tion of morphine and oxycodone dramatically decreased and increased between 2005 and 2016, respectively; however the annual number of related $\mathrm{AE}$ reports per $\mathrm{kg}$ consumption hovered between $0.1-0.2$. By contrast, the annual consumption of fentanyl dramatically increased between 2005 and 2009, peaked in 2009, but gradually declined thereafter. However, the number of $\mathrm{AE}$ cases per $\mathrm{kg}$ of fentanyl increased from 2009 , exceeding 0.05 in 2012 , but subsequently declined to approximately 0.04 .

Temporal Changes in Outcome "Death" in OpioidRelated AE Reports Between April 2004 and March 2017, we retrieved 335 cases $(10.9 \%)$ of opioid-related AE reports in which the outcome was "death." The number and rate were higher for fentanyl, intermediate for morphine, and lower for oxycodone, as shown in Table 2. 
Table 2. Adverse Event (AE) Reports with the Outcome "Death" and Reported Odds Ratio for Each Opioid

\begin{tabular}{|c|c|c|c|c|}
\hline & AE reports (number) & Outcome “death" reports (number (\%)) & ROR $(95 \% \mathrm{CI})$ & $p$ Value \\
\hline Total opioids & 3042 & $335(10.9 \%)$ & $1.50(1.34-1.68)$ & $<0.0001$ \\
\hline Morphine & 579 & $65(11.2 \%)$ & $1.54(1.19-2.00)$ & 0.0021 \\
\hline Oxycodone & 789 & $49(6.2 \%)$ & $0.81(0.61-1.08)$ & 0.140 \\
\hline Fentanyl & 1481 & $191(12.9 \%)$ & $1.80(1.55-2.10)$ & $<0.0001$ \\
\hline Other opioids & 193 & $30(15.5 \%)$ & $2.23(1.51-3.29)$ & $<0.0001$ \\
\hline
\end{tabular}

ROR: reported odds ratio, CI: confidential interval.
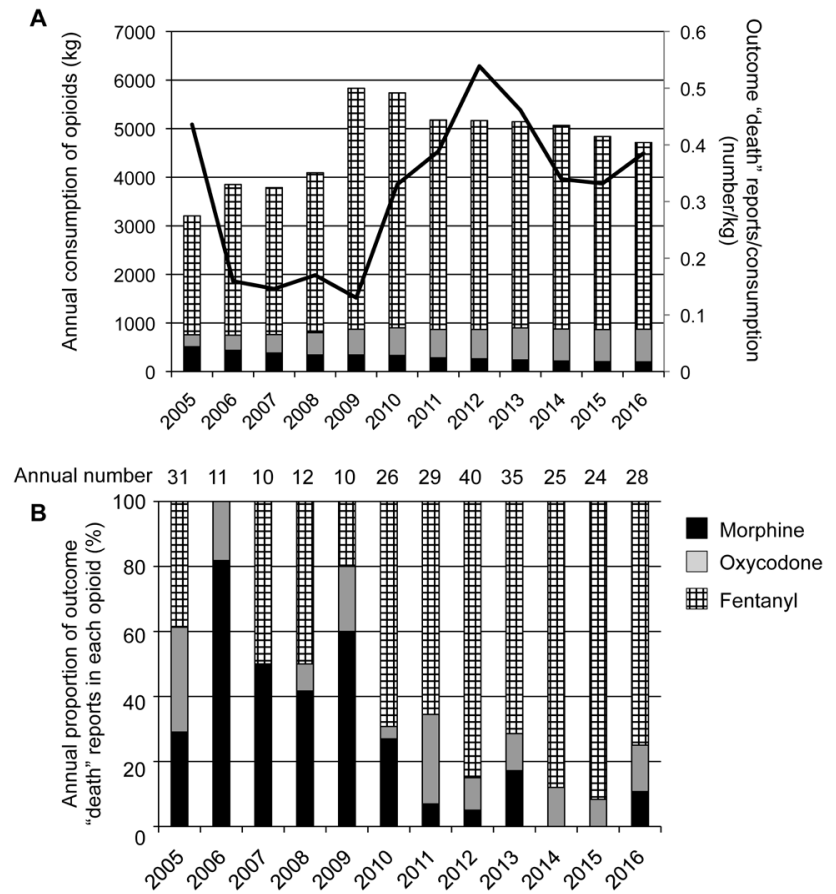

Fig. 3. Changes in the Annual Number of Adverse Event (AE) Reports with the Outcome "Death" between 2005 and 2016

(A) The left axis (bar graph) represents the annual consumption (in morphineequivalent doses, $\mathrm{kg}$ ) of morphine, oxycodone and fentanyl, as indicated. The right axis (line graph) represents the annual number of opioid-related AE reports with the outcome "death" according to the annual consumption (in morphine-equivalent doses, $\mathrm{kg}$ ). (B) Graph showing the annual proportion of AE reports with the outcome "death" for each opioid (morphine, oxycodone, and fentanyl), as indicated, among the total number of reports with this outcome.

The annual number and number per consumption of outcome "death" in all opioid-related AE reports were large in 2005. Although these declined between 2006 and 2009, they increased again from 2010 onwards (Fig. 3A). The rate of outcome "death" due to morphine AEs was high, representing a large proportion of outcome "death" reports by opioids between 2005 and 2010, while it dramatically declined from 2011 onwards. Instead, the rate of outcome "death" due to fentanyl gradually increased from 2005, representing the highest proportion since 2010. By contrast, the rate of outcome "death" due to oxycodone were relatively few during the survey period (Fig. 3B). The calculated ROR for the outcome "death" in opioid-related AEs was 1.50. The RORs for morphine and fentanyl were 1.54 and 1.80 , respectively, which were statistically significant. The ROR for oxycodone was 0.81 , which was not statistically significant (Table 2).

Among 191 fentanyl-related AE reports with outcome "death," we eliminated the reports which were unlikely to be recognized as fentanyl-related, such as those including "death," "malignant neoplasm," and "aggravation of underlying diagnosis" as AEs. The most frequent AEs were respiratory symptoms, including respiratory depression (3.7\%), respiratory impairment $(3.1 \%)$, and interstitial lung disease $(1.6 \%$; Table 3). Similarly, morphine- and oxycodone-related AEs with outcome "death" were also analyzed. The AEs reported more than two cases were kidney impairment (10.8\%), aspiration pneumonitis $(6.2 \%)$, respiratory depression $(4.6 \%)$, and respiratory impairment (4.6\%) in morphine and interstitial lung disease $(8.2 \%)$, aspiration pneumonitis $(6.1 \%)$ in oxycodone.

\section{DISCUSSION}

In this study, we found that deaths were frequently reported as the outcome following opioid-related AEs. Although the causes of death for the reported cases cannot be determined by JADER analysis, the JADER database includes case records of death as the result of opioid-related serious AEs. They can occur in patients treated with opioids even when adequate caution is paid. Thus, it is important that pharmacists engaging in palliative care reduce opioid-related deaths by promoting appropriate use of opioids.

Between April 2004 and March 2017, 3042 incidents of opioid-related AEs were reported in the JADER database. In this period, opioid-related AE reports increased, while their percentage among all AE reports in JADER remained stable; thus, the incidence did not dramatically change. The increased number of AE reports is likely due to the overall increase of AE cases reported by physicians and other medical personnel. Moreover, the total annual consumption of opioids in Japan has also increased over this period. ${ }^{11)}$ Taken together, these evidences suggest that the annual incidence of opioid-related AEs has not dramatically increased despite the increased consumption of opioids. Thus, it is seems that opioid-induced AEs are being appropriately managed by the medical personnel in Japan.

Opioid-related serious AEs, such as delirium, respiratory depression, and altered/decreased conscious level, were frequently reported in JADER. By contrast, general AEs induced by opioids, such as nausea, vomiting, somnolence, and constipation, were only occasionally reported. Although opioids are used to alleviate cancer and non-cancer pain, the risk of serious AEs gradually increased in a manner dependent on increased opioid doses, ${ }^{12,13)}$ and opioid overdose may cause lethal AEs, such as respiratory depression. ${ }^{14)}$ It is reported that oral morphine titration in cancer patients is useful to achieve rapid pain control and is well tolerated, ${ }^{15)}$ and mild to moderate AEs induced by morphine could be controlled by reducing the morphine dose by 25 to $50 \%{ }^{16)}$ Clinical guideline published by European Society for Medical Oncology recommended 
Table 3. Ranking of the Adverse Events Reported for Fentanyl with the Outcome "Death"

\begin{tabular}{lc}
\hline \multicolumn{1}{c}{ Side effect } & Reported number (ratio) \\
\hline Dead & $12(6.3 \%)$ \\
Malignant neoplasm & $12(6.3 \%)$ \\
Malignant neoplasm of lung & $9(4.7 \%)$ \\
Developed malignant neoplasm & $8(4.2 \%)$ \\
Respiratory depression & $7(3.7 \%)$ \\
Respiratory impairment & $6(3.1 \%)$ \\
Stomach cancer & $5(2.6 \%)$ \\
Decrease of awareness level & $5(2.6 \%)$ \\
Multiple organ failure & $5(2.6 \%)$ \\
Sepsis & $5(2.6 \%)$ \\
Pancreatic cancer & $4(2.1 \%)$ \\
Cardiac failure & $4(2.1 \%)$ \\
Pneumoniae & $3(1.6 \%)$ \\
Interstitial lung disease & $3(1.6 \%)$ \\
Colon cancer & $3(1.6 \%)$ \\
Developed underlying disease & $3(1.6 \%)$ \\
Cardiac arrest & $3(1.6 \%)$ \\
Toxic epidermal necrosis & $3(1.6 \%)$ \\
Intestinal trephination & $3(1.6 \%)$ \\
\hline
\end{tabular}

that the management of opioid-induced adverse effects is an important aspect of pain management because each adverse effect requires a careful assessment and treatment strategy. ${ }^{17)}$ Therefore, opioid dose must be carefully adjusted depending on individual circumstances with continuous monitoring for effectiveness and avoidance of overdose.

In Japan, several formulations of opioids were marketed during the survey period, 2004-2017, including a morphine internal formulation marketed in 2003, fast-acting oxycodone in 2007, two types of transdermal fentanyl in 2008 and 2010, an oxycodone injection drug in 2012, and fentanyl sublingual and buccal tablets in 2014. Marketing of novel opioid formulations can affect consumption and shared components of opioids, which may lead to changes in the number, incidence, or kind of opioid-related AEs. Our analysis showed that the annual consumption of morphine decreased and that of oxycodone increased during the survey period, while there were no dramatic changes in the annual incidence of their AEs. Thus, the reported incidence of AEs for these two drugs was almost constant despite the changes in their annual consumption. The annual incidence of fentanyl-related AEs was lower than that of morphine and oxycodone, which may be due to the fewer common AEs of the transdermal fentanyl patch, such as nausea, vomiting, somnolence, and constipation, than those of morphine and oxycodone. ${ }^{18,19)}$ Nevertheless, the annual incidence of fentanyl-related AEs increased since 2010, although it was still lower than that of morphine and oxycodone. Interestingly, the rate of outcome "death" following fentanyl-related AEs became highest since 2010 and was 5-10 times higher than that following morphine- and oxycodone-related AEs since 2012. Until 2009 in Japan, the indication of transdermal fentanyl patches was limited to treat moderate-to-severe cancer pain. However, in 2010, the indication was expanded to both cancer and non-cancer chronic pain. In addition, the one-day transdermal fentanyl patch was approved in 2011 and sublingual and buccal tablets in 2013. Thus, it is possible that the indication expansion and novel formulations of fentanyl may have increased the annual incidence of related AEs and death. The ROR of fentanyl (1.80) was slightly higher than that of morphine (1.54), although there was a small difference in the percentage of AE reports with the outcome "death" between the two drugs (fentanyl: $12.9 \%$ and morphine: $11.2 \%$ ). The large number of fentanyl-related deaths may be due only to its large consumption. Moreover, the risk of "death" as an outcome of fentanyl-related AE is higher than that in the case of morphine and oxycodone, despite the smaller number of fentanyl-related AE reports per its consumption. The present findings indicate that this number and the risk of outcome "death" in Japan would be higher for fentanyl than for morphine and oxycodone. Alternatively, it is also suggested that extra cautions should be paid for morphine- and oxycodonerelated $\mathrm{AE}$ incidence other than death.

The most frequent fentanyl-related AEs with outcome "death" were "respiratory depression" and "respiratory impairment," after excluding those unlikely to be related with fentanyl, such as "death" or "malignant neoplasm." Similarly, AEs associated with respiratory depression were also detected as the cause of outcome "death" in morphine- and oxycodonerelated AEs. These results of the present study suggest that respiratory depression should be considered a common $\mathrm{AE}$ of opioids leading to death. The risk for respiratory depression due to fentanyl, which is directly linked to death, ${ }^{20)}$ has been frequently reported. ${ }^{21-23)}$ Therefore, caution should be certainly paid for ensuring the appropriate use of fentanyl. Nevertheless, in the past 10 years, respiratory depression leading to deaths related with fentanyl has been sometimes reported. Fentanyl is a very convenient drug used in palliative care, because it can be administered transdermally and is available in sublingual and buccal tablets, allowing rapid relief of breakthrough pain. Thus, further countermeasures should be developed and promoted. Between 2001 and 2016, the number of opioid-related deaths in the U.S. markedly increased by $345 \%$, from 9489 to 42445 deaths. ${ }^{24)}$ In contrast, in the survey period of this study, the number of opioid-related AEs leading to outcome "death" did not increase in Japan. There were only 28 such cases in 2016, which was less than 1/1000 of the number in the U.S., even if the number in Japan is underestimated. Although there are some differences between the two countries (e.g., population, number of patients prescribed opioids, and opioid consumption), the number of opioid-related deaths in Japan can remain lower than that in the U.S.

This study has certain limitations. As our analysis was based on self-reporting, we could not verify the causal relationship between a medication and AEs or outcome. For this reason, the present data should be viewed with caution. Furthermore, the reported AEs included events such as "aggravation of underlying diagnosis" and "malignant neoplasm," which are unlikely to be caused by the drugs. Further investigations will be needed to collect specific information regarding deaths connected to opioid-related AEs. In the present study, we could not directly compare the data between Japan and other countries. Most reports in North America and other Western countries show the number of AEs adjusted by the number of patients, such as number of patients per 100000 . However, we could not apply the data in relation to the population of patients prescribed opioids in Japan, due to the lack of such data in the JADER database. Therefore, the relationship between the number of opioid-related $\mathrm{AE}$ and those of 
patients prescribed opioids in Japan could not be analyzed in this study.

In conclusion, we analyzed the changes in the annual number of opioid-related AE and deaths reported during a 12-year period and included in the JADER database. Although the number of deaths resulting from fentanyl-related AEs seems to be higher than that of morphine or oxycodone in Japan, there is currently no evidence for any dramatic increase in the incidence of opioid-related AEs. This is the first study in Japan to suggest that opioid-related AEs can be controlled through proper monitoring and management by medical personnel. We hope that our findings will be channeled back to society as one step toward greater public awareness.

Acknowledgments We thank the members of the Japanese Society for Pharmaceutical Palliative Care and Sciences who cooperated in this study.

Conflict of Interest The authors declare no conflict of interest.

\section{REFERENCES}

1) Herzig SJ, Rothberg MB, Cheung M, Ngo LH, Marcantonio ER. Opioid utilization and opioid-related adverse events in nonsurgical patients in U.S. hospitals. J. Hosp. Med., 9, 73-81 (2014).

2) Dart RC, Surratt HL, Cicero TJ, Parrino MW, Severtson SG, Bucher-Bartelson B, Green JL. Trends in opioid analgesic abuse and mortality in the United States. N. Engl. J. Med., 372, 241-248 (2015).

3) Kuehn BM. Safety plan for opioids meets resistance: opioid-linked deaths continue to soar. JAMA, 303, 495-497 (2010).

4) Fischer B, Rehm J. Deaths related to the use of prescription opioids. CMAJ, 181, 881-882 (2009).

5) Dhalla IA, Mamdani MM, Sivilotti ML, Kopp A, Qureshi O, Juurlink DN. Prescribing of opioid analgesics and related mortality before and after the introduction of long-acting oxycodone. CMAJ, 181, 891-896 (2009).

6) Madadi P, Hildebrandt D, Lauwers AE, Koren G. Characteristics of opioid-users whose death was related to opioid-toxicity: a population-based study in Ontario, Canada. PLOS ONE, 8, e60600 (2013).

7) Duthey B, Scholten W. Adequacy of opioid analgesic consumption at country, global, and regional levels in 2010, its relationship with development level, and changes compared with 2006. J. Pain Symptom Manage., 47, 283-297 (2014).

8) Okie S. A flood of opioids, a rising tide of deaths. N. Engl. J. Med., 363, 1981-1985 (2010).

9) Hirooka T, Ymada M. Evaluation of risk of adverse reaction using PMDA 'Adverse Drug Reaction Database.' Database SAS User Groups Ronbun-syu, 263-270 (2012).

10) Nagai J, Uesawa Y, Kagaya H. Analyses of oxycodone-induced adverse effects based on the Japanese Adverse Drug Event Report
Database. Palliative Care Research, 10, 161-168 (2015).

11) Foundation for Promotion of Cancer Research. "CANCER STATISTICS JAPAN 2017.”: 〈https://ganjoho.jp/data/reg_stat/statistics/brochure/2017/cancer_statistics_2017.pdf), cited March, 2018.

12) Zedler B, Xie L, Wang L, Joyce A, Vick C, Kariburyo F, Rajan P, Baser O, Murrelle L. Risk factors for serious prescription opioidrelated toxicity or overdose among Veterans Health Administration patients. Pain Med., 15, 1911-1929 (2014).

13) Bohnert AS, Valenstein M, Bair MJ, Ganoczy D, McCarthy JF, Ilgen MA, Blow FC. Association between opioid prescribing patterns and opioid overdose-related deaths. JAMA, 305, 1315-1321 (2011).

14) Barletta JF. Clinical and economic burden of opioid use for postsurgical pain: focus on ventilatory impairment and ileus. Pharmacotherapy, 32 (Suppl.), 12S-18S (2012).

15) De Conno F, Ripamonti C, Fagnoni E, Brunelli C, Luzzani M, Maltoni M, Arcuri E, Bertetto O; MERITO Study Group. The MERITO Study: a multicentre trial of the analgesic effect and tolerability of normal-release oral morphine during 'titration phase' in patients with cancer pain. Palliat. Med., 22, 214-221 (2008).

16) Cherny N, Ripamonti C, Pereira J, Davis C, Fallon M, McQuay H, Mercadante S, Pasternak G, Ventafridda V, Expert Working Group of the European Association of Palliative Care Network. Strategies to manage the adverse effects of oral morphine: an evidence-based report. J. Clin. Oncol., 19, 2542-2554 (2001).

17) Fallon M, Giusti R, Aielli F, Hoskin P, Rolke R, Sharma M, Ripamonti CI, ESMO Guidelines Committee. Management of cancer pain in adult patients: ESMO Clinical Practice Guidelines. Ann. Oncol., 29 (Suppl. 4), iv166-iv191 (2018).

18) Mystakidou K, Tsilika E, Parpa E, Kouloulias V, Kouvaris I, Georgaki S, Vlahos L. Long-term cancer pain management in morphine pre-treated and opioid naive patients with transdermal fentanyl. Int. J. Cancer, 107, 486-492 (2003).

19) Donner B, Zenz M, Tryba M, Strumpf M. Direct conversion from oral morphine to transdermal fentanyl: a multicenter study in patients with cancer pain. Pain, 64, 527-534 (1996).

20) Jumbelic MI. Deaths with transdermal fentanyl patches. Am. J. Forensic Med. Pathol., 31, 18-21 (2010).

21) Nadpara PA, Joyce AR, Murrelle EL, Carroll NW, Carroll NV, Barnard M, Zedler B. Risk factors for serious prescription opioidinduced respiratory depression or overdose: comparison of commercially insured and veterans health affairs populations. Pain Med., 19, 79-96 (2018).

22) Fox LM, Hoffman RS, Vlahov D, Manini AF. Risk factors for severe respiratory depression from prescription opioid overdose. $A d$ diction, 113, 59-66 (2018).

23) Dahan A, Yassen A, Bijl H, Romberg R, Sarton E, Teppema L, Olofsen E, Danhof M. Comparison of the respiratory effects of intravenous buprenorphine and fentanyl in humans and rats. Br. J. Anaesth., 94, 825-834 (2005).

24) Gomes T, Tadrous M, Mamdani MM, Paterson JM, Juurlink DN. The burden of opioid-related mortality in the United States. JAMA Netw. Open, 1, e180217 (2018). 